COMMUNICATIONS IN

ANALYSIS AND GEOMETRY

Volume 12, Number 1, 305-320, 2004

\title{
On Dimension Reduction in the Kähler-Ricci Flow
}

\author{
Huai-Dong CAO
}

\begin{abstract}
We extend the method of dimension reduction of Hamilton for the Ricci flow to the Kähler-Ricci flow. In the case of complex dimension $n=2$, we prove a dimension reduction theorem for complete translating Kähler-Ricci solitons with nonnegative bisectional curvature. For $n>2$, we also prove a dimension reduction theorem for complete ancient solutions of the Kähler-Ricci flow with nonnegative bisectional curvature under a finiteness assumption on the Chern number $c_{1}^{n}$.
\end{abstract}

\section{Introduction.}

In minimal surface theory and harmonic map theory, there is a well-known general principle of dimension reduction which is very useful in studying singularities. The basic idea is that one first takes a limit of a sequence of dilations to model a singularity (blow-up), and then takes a sequence of origin going out to infinity and shrinking back down (blow-down) to get a new limit of lower dimension. In [13], Richard Hamilton showed us how to carry out this general idea of dimension reduction to solutions of the Ricci flow on Riemannian manifolds with nonnegative curvature operator and local injectivity radius estimate (cf. Section 22 of [13]), and used it to prove important results about singularity formations of the Ricci flow. See also the recent work of Chen-Tang-Zhu [6] for a very nice application of Hamilton's dimension reduction result to the Kähler-Ricci flow on noncompact complex surfaces with positive bisectional curvature and maximal volume growth. For studying formations of singularities of the Kähler-Ricci flow in complex dimension $n \geq 2$, it has been very desirable but a challenge to extend Hamilton's dimension reduction for the Ricci flow to solutions of the Kähler-Ricci flow with positive holomorphic bisectional curvature, a weaker assumption than nonnegative curvature operator.

In the proof of Hamilton's dimension reduction result for the Ricci flow, the assumption of nonnegative curvature operator is used in an essential way

\footnotetext{
${ }^{1}$ Research supported in part by NSF grant DMS-0206847.
} 
in both finding a flat curvature direction of the blow-down limit, and the splitting of flat factors. In the Kähler case, the splitting theorem for complete solutions of the Kähler-Ricci flow with nonnegative bisectional curvature (cf. Theorem 2.1) was observed by the author back in mid 80's. The main difficulty has been how to produce a flat direction in blow-down limits to allow the splitting when we only have nonnegative bisectional curvature. In this paper we resolve this difficulty completely in the case of of complex dimension $n=2$ and show how the dimension reduction of Hamilton for the Ricci flow can be extended to the Kähler case for 2-dimensional translating Kähler-Ricci solitons (cf. Theorem 1.1). For $n>2$, we also prove a dimension reduction result for ancient solutions of the Kähler-Ricci flow with nonnegative bisectional curvature under a certain finiteness assumption of the Chern number integral $c_{1}^{n}$ (Theorem 1.2). When $n=2$, we are able to show that the second Chern number integral is well defined (cf. Proposition 4.1) for translating Kähler-Ricci solitons of nonnegative bisectional curvature. By exploring the relation between the integrands of the Chern numbers $c_{1}^{2}$ and $c_{2}$, we show that $c_{1}^{2}$ is automatically bounded.

A complete solution to the Kähler-Ricci flow

$$
\frac{\partial}{\partial t} g_{i \bar{j}}(x, t)=-R_{i \bar{j}}(x, t)
$$

is a time-dependent family of complete Kähler metrics $g=\sum g_{i \bar{j}}(x, t) d z^{i} d z^{\bar{j}}$ on a complex manifold $X^{n}$, either compact or noncompact, and some time interval satisfying Eq. (1), where $R_{i \bar{j}}(x, t)$ denotes the Ricci tensor of the metric $g$ at time $t$.

Definition (cf. Section 16 of [13]) A solution $g$ to the Kähler-Ricci flow (1), where either the complex manifold $X$ is compact or at each time $t$ the metric $g$ is complete with bounded and nonnegative bisectional curvature, is called a singularity model or limit solution of Eq.(1) if it is non flat and of one of the following three types:

Type I: $X$ is either compact or noncompact and $g$ exists on an ancient time interval $-\infty<t<\Omega$ for some constant $\Omega$ with $0<\Omega<\infty$, and the scalar curvature $R$ satisfies the inequality

$$
R(x, t) \leq \Omega /(\Omega-t)
$$

everywhere with equality at some origin point $O$ at time $t=0$.

Type II : $X$ is noncompact and $g$ exists on the eternal time interval $-\infty<t<+\infty$, and

$$
R(x, t) \leq 1
$$


everywhere with equality at some origin point $O$ at time $t=0$.

Type I and Type II singularity models above typically arise as limit of blowups of maximal solutions to Eq.(1) of the corresponding type. We remark that Type II limit solutions of nonnegative bisectional curvature are necessarily translating Kähler-Ricci solitons by our previous work [3] (see Prop. 3.1). Our first result is the following dimension reduction theorem for 2dimensional Type II singularity models:

Theorem 1.1 Let $g$ be a Type II singularity model, or equivalently a translating Kähler-Ricci soliton, of the Kähler-Ricci flow (1) on a noncompact 2dimensional complex surface $X^{2}$ and the eternal time interval $-\infty<t<\infty$, complete with bounded and nonnegative bisectional curvature at each time. If $g$ satisfies the local injectivity radius estimate

$$
\operatorname{inj}_{X}(x, t) \geq \beta / \sqrt{R(x, t)}
$$

for all $x \in X^{2}$ and some constant $\beta>0$ independent of $x$, then there exists a sequences of dilations of $g$ which converges to a limit $\widehat{g}$, which is again a complete solution to the Kähler-Ricci flow (1) on some noncompact complex surface $\widehat{X}^{2}$ and ancient time interval $-\infty<t<\Omega$, with nonnegative bisectional curvature. Moreover, $\left(\widehat{X}^{2}, \widehat{g}\right)$ splits as a quotient of the product $\mathbf{C} \times \mathbf{C P}^{1}$ of flat complex plane $\mathbf{C}$ and the complex projective plane $\mathbf{C P}^{1}$.

When a complete noncompact Riemannian manifold $\left(M^{m}, g\right)$ of real dimension $m$ has nonnegative Ricci curvature, the ratio $V\left(B_{s}\right) / s^{m}$, where $V\left(B_{s}\right)$ is the volume of geodesic ball of radius $s$ centered at some origin point $O \in M$, is monotone decreasing in $s$ by the volume comparison theorem. So one can consider the asymptotic volume ratio (cf. Hamilton [13], P.74)

$$
\nu_{M}=\lim _{s \rightarrow \infty} V\left(B_{s}\right) / s^{m} .
$$

The definition of $\nu_{M}$ is independent of the choice of the origin $O$. Note that $\nu_{M}>0$ if and only if $\left(M^{m}, g\right)$ has Euclidean volume growth.

Corollary 1.1 There exists no 2-dimensional Type II singularity models with bounded and nonnegative bisectional curvature, and with Euclidean volume growth. In other words, the asymptotic volume ratio $\nu$ of any 2-dimensional Type II singularity model with nonegative bisectional curvature must be zero.

Remark 1: Corollary 1.1 was first proved by Chen-Zhu [7].

Our second result is a dimension reduction for ancient solutions:

Theorem 1.2 Let $g$ be a complete ancient solution to the Kähler-Ricci flow on a noncompact complex manifold $X^{n}(n \geq 2)$ and time interval $-\infty<$ 
$t<T(0<T \leq \infty)$, with bounded and nonnegative bisectional curvature, and positive Ricci curvature at each time. Assume that the following conditions are satisfied:

(i) Finite asymptotic scalar curvature ratio (ASCR):

$$
A \equiv \limsup _{s \rightarrow \infty} R s^{2}=\infty
$$

at $t=0$. Here $s$ denotes the distance function on $X$ from some fixed origin point;

(ii) Local injectivity radius estimate: there exists a constant $\beta>0$ such that $\forall x \in X^{n}$ and $t$,

$$
\operatorname{inj}_{X}(x, t) \geq \beta / \sqrt{R(x, t)}
$$

(iii) Finite Chern number: $c_{1}^{n}(X) \equiv \int_{X} R c^{n}<\infty$.

Then there exists a sequences of dilations of $g$ which converges to a limit $\widehat{g}$, which is again a complete solution to the Kähler-Ricci flow (1) on some noncompact complex manifold $\widehat{X}^{n}$ and ancient time interval $-\infty<t<\Omega$, with nonnegative bisectional curvature. Moreover, the limit $\left(\widehat{X}^{n}, \widehat{g}\right)$ splits as a quotient of a product $\mathbf{C}^{\mathbf{k}} \times \widehat{N}^{n-k}$ with $k \geq 1$ flat in the direction of $\mathbf{C}^{k}$, and where the interesting factor $\widehat{N}^{n-k}$ has positive Ricci curvature, and is either a Type I limit solution, or Type II limit solution with $c_{1}^{n-k}(\widehat{N})=\infty$.

Remark 2: We conjecture that the only possible factor $\widehat{N}$ in Theorem 1.2 is of Type I. Moreover, we conjecture that there is no noncompact Type I limit solutions with nonnegative bisectional curvatue and positive Ricci curvature. Note that Hamilton (Section 26 of [13]) proved that in complex dimension one, the only Type I ancient solution is either the complex projective plane $\mathbf{C P}^{1}$, or flat complex plane $\mathbf{C}$ and its quotients.

Remark 3: If $g$ is a Type I or Type II singularity model, then assumption (i) in Theorem 1.2 holds automatically. Note also that if $g$ comes as a blow-up limit of a solution to the Kähler-Ricci flow (1) on a compact Kähler manifold then condition (iii) in Theorem 1.2 is automatically satisfied.

Acknowledgment: The research was carried out while the author was on leave to the Institute for Pure and Applied Mathematics (IPAM) at UCLA. Part of the paper was written up when he attended the five-week long summer workshop on Geometric Evolution Equations at National Center of Theoretical Sciences (NCTS) in Hsinchu, Taiwan in 2002. The author would like to thank both IPAM and NCTS for their hospitality and financial support. 


\section{A splitting theorem for the Kähler-Ricci flow.}

In this section we state a splitting theorem for solutions of the Kähler-Ricci flow (1) with nonnegative bisectional curvature. The result is a natural analogue to the splitting theorem of Hamilton [9] for solutions of the Ricci flow on Riemannian manifolds with nonnegative curvature operator. This splitting theorem will be useful in subsequent sections, in particular in the proof of our dimension reduction theorems.

Theorem 2.1 Let $g$ be a complete solution of the Kähler-Ricci flow (1) on a noncompact simply connected complex manifold $X^{n}$ of dimension $n$ and some open time interval $I$, with bounded and nonnegative holomorphic bisectioanl curvature. Then either $g$ is of positive Ricci curvature for all $x \in X$ and all $t \in I$, or $(X, g)$ splits holomorphically isometrically into a product $C^{k} \times N^{n-k}(k \geq 1)$ flat in $C^{k}$ direction and $N$ being of nonnegative holomorphic bisectioanl curvature and positive Ricci curvature.

Proof: The proof can proceed essentially along the same line as in [9], except one needs to use strong maximum principle for noncompact manifolds similar to those in $[11,12]$. For simplicity, we only present the proof in the compact case.

First we claim that for any $t_{0}$, there exists a time interval $t_{0}<t<t_{0}+\delta$, on which the rank of the Ricci tensor $R_{i \bar{j}}$ is constant and the null space of $R_{i \bar{j}}$ is invariant under parallel translation and invariant in time. First, recall that the Ricci tensor satisfies the evolution equation:

$$
\frac{\partial}{\partial t} R_{i \bar{j}}=\Delta R_{i \bar{j}}+\Phi_{i \bar{j}}
$$

where $\Phi_{i \bar{j}}=R_{i \bar{j} k \bar{l}} R_{l \bar{k}}$. Let $0 \leq \sigma_{1} \leq \sigma_{2} \leq \cdots \leq \sigma_{n}$ be the eigenvalues of the Ricci tensor. Then $\sigma_{1}+\cdots+\sigma_{k}$ is a concave function of $R_{i \bar{j}}$ and is invariant under parallel translation, since

$$
\sigma_{1}+\cdots+\sigma_{k}=\inf \left\{\operatorname{tr}\left(R_{i \bar{j}} \mid E: E \subset T_{X} \text { is any subspace of } \operatorname{dim} \mathrm{k}\right\} .\right.
$$

Note that dim of the null space of $R_{i \bar{j}}$ is $\geq k$ if and only if $\sigma_{1}+\cdots+\sigma_{k}=0$. If $\sigma_{1}+\cdots+\sigma_{k}>0$ at one point at $t=t_{0}$, then by the strong maximum principle, it is positive everywhere for all $t>t_{0}$. So it follows that rank of $R_{i \bar{j}}$ remains constant on some time interval $t_{0}<t<t_{0}+\delta$.

Next, let $v$ be any smooth section of the holomorphic tangent bundle $T_{X}$ in the null space of $R_{i \bar{j}}$ on $t_{0}<t<t_{0}+\delta$. Then

$$
0=\frac{\partial}{\partial t}\left(R_{i \bar{j}} v^{i} v^{\bar{j}}\right)=\left(\frac{\partial}{\partial t} R_{i \bar{j}}\right) v^{i} v^{\bar{j}}+R_{i \bar{j}}\left(\frac{\partial v^{i}}{\partial t} v^{\bar{j}}+v^{i} \frac{\partial v^{\bar{j}}}{\partial t}\right)
$$


Since $R_{i \bar{j}} v^{i}=R_{i \bar{j}} v^{\bar{j}}=0$, the last term vanishes. Also

$$
\begin{aligned}
0= & \Delta\left(R_{i \bar{j}} v^{i} v^{\bar{j}}\right)=\left(\Delta R_{i \bar{j}}\right) v^{i} v^{\bar{j}}+D_{k} R_{i \bar{j}}\left(v^{\bar{j}} D_{\bar{k}} v^{i}+v^{i} D_{\bar{k}} v^{\bar{j}}\right) \\
& +D_{\bar{k}} R_{i \bar{j}}\left(v^{\bar{j}} D_{k} v^{i}+v^{i} D_{k} v^{\bar{j}}\right)+R_{i \bar{j}}\left(D_{k} v^{i} D_{\bar{k}} v^{\bar{j}}+D_{\bar{k}} v^{i} D_{k} v^{\bar{j}}\right) \\
& +R_{i \bar{j}}\left(v^{\bar{j}} \Delta v^{i}+v^{i} \Delta v^{\bar{j}}\right)
\end{aligned}
$$

and again the last term disappears. Since

$$
0=D_{k}\left(R_{i \bar{j}} v^{i}\right)=\left(D_{k} R i \bar{j}\right) v^{i}+R_{i \bar{j}} D_{k} v^{i},
$$

and

$$
0=D_{k}\left(R_{i \bar{j}} v^{\bar{j}}\right)=\left(D_{k} R i \bar{j}\right) v^{\bar{j}}+R_{i \bar{j}} D_{k} v^{\bar{j}},
$$

etc., we get from the evolution equation (3)

$$
R_{i \bar{j}}\left(D_{k} v^{i} D_{\bar{k}} v^{\bar{j}}+D_{\bar{k}} v^{i} D_{k} v^{\bar{j}}\right)+\Phi_{i \bar{j}} v^{i} v^{\bar{j}}=0 .
$$

Since $R_{i \bar{j}} \geq 0$ and $\Phi_{i \bar{j}}=R_{i \bar{j} k \bar{l}} R_{l \bar{k}} \geq 0$, we must have $v$ also in the null space of $\Phi_{i \bar{j}}$ and $D_{k} v^{i}, D_{k} v^{\bar{j}}$ etc in the null spaces of $R_{i \bar{j}}$ for all $\mathrm{k}$. This shows that the null space of the Ricci tensor is invariant under parallel translation and $\operatorname{null}\left(R_{i \bar{j}}\right) \subset \operatorname{null}\left(\Phi_{i \bar{j}}\right)$.

Finally, to see the null space of the Ricci tensor is also invariant in time, note first that $\Delta v^{i}$ and $\Delta v^{j}$ lies in the null space of the Ricci tensor. Then

$$
0=D_{k}\left(R_{i \bar{j}} D_{\bar{k}} v^{i}\right)=D_{k} R_{i \bar{j}} D_{\bar{k}} v^{i}+R_{i \bar{j}} D_{k} D_{\bar{k}} v^{i}
$$

and

$$
0=D_{\bar{k}}\left(R_{i \bar{j}} D_{k} v^{i}\right)=D_{\bar{k}} R_{i \bar{j}} D_{k} v^{i}+R_{i \bar{j}} D_{\bar{k}} D_{k} v^{i}
$$

and so

$$
D_{k} R_{i \bar{j}} D_{\bar{k}} v^{i}+D_{\bar{k}} R_{i \bar{j}} D_{k} v^{i}=0
$$

Then

$$
0=\Delta\left(R_{i \bar{j}} v^{i}\right)=\left(\Delta R_{i \bar{j}}\right) v^{i}+\frac{1}{2}\left(D_{k} R_{i \bar{j}} D_{\bar{k}} v^{i}+D_{\bar{k}} R_{i \bar{j}} D_{k} v^{i}\right)
$$

and hence $\left(\Delta R_{i \bar{j}}\right) v^{i}=0$. Then

$$
0=\frac{\partial}{\partial t}\left(R_{i \bar{j}} v^{i}\right)=R_{i \bar{j}} \frac{\partial v^{i}}{\partial t}+\left(\Delta R_{i \bar{j}}+R_{i \bar{j} k \bar{l}} R_{l \bar{k}}\right) v^{i} .
$$

Now $R_{i \bar{j} k \bar{l}} R_{l \bar{k}} v^{i}=0$ whenever $v$ is in the null space of $R_{i \bar{j}}$. Thus $R_{i \bar{j}} v^{i}=0$, and $\partial v / \partial t$ lies in the null space of the Ricci tensor as well. This shows the 
null space of the Ricci tensor is invariant in time. Therefore, either the null space of the Ricci tensor is trivial, or, by the De Rham decomposition theorem (see e.g. Theorem 8.1 in [14]) and induction on the dimension of the null space, the underlying complex manifold $X$ splits holomorphically isometrically into a product of some flat complex Euclidean space $\mathbf{C}^{k}$ and another factor $N^{n-k}$ whose Ricci tensor is everywhere positive. This completes the proof of Theorem 2.1.

Remark: A similar argument of the proof has been used recently by $\mathrm{Ni}$ Tam in their work [16] on a Liouville type theorem for plurisubharmonic functions.

\section{Type II singularity models and Kähler-Ricci solitons.}

In this section, we collect some known results about Type II singularity models and translating Kähler-Ricci solitons.

An important class of Type II singularity models is given by translating Kähler-Ricci soliton (KRS), which is an eternal solution $g$ moving along the Kähler-Ricci flow (1) under a one-parameter family of biholomorphisms of a noncompact complex manifold $X$ generated by some holomorphic vector field $V$ on $X$. That is we have $g(t)=\phi^{*}(t) g(o)$, where $\phi(t)=\exp \{-t V\}$ is the one-parameter family of automorphisms of $X$. Equivalently, for each $t$, we have

$$
R_{i \bar{j}}=L_{V} g_{i \bar{j}},
$$

the Lie derivative of $g$ in the direction of $V$. Thus in local holomorphic coordinates, translating KRSs are characterized by equations

$$
R_{i \bar{j}}=D_{\bar{j}} V_{i}=D_{i} V_{\bar{j}}, \quad \text { and } \quad D_{j} V_{i}=D_{\bar{j}} V_{\bar{i}}=0 .
$$

Note that the condition $D_{j} V_{i}=0$ is equivalent to saying that the vector field $V$ is holomorphic. If the vector field $V$ is the gradient of a real-valued smooth function $f$ on $X$ so that $V^{i}=g^{i \bar{j}} \partial_{j} f$, then we call $g$ a gradient translating $K R S$ and $f$ a potential function of the soliton. In this case, the above soliton equation becomes

$$
R_{i \bar{j}}=D_{i} D_{\bar{j}} f, \quad \text { and } \quad D_{i} D_{j} f=0 .
$$

It turns out all Type II singularity models with nonnegative curvature arise this way:

Proposition 3.1 (Cao [3]) Any Type II singularity model $(X, g)$ of the Kähler-Ricci flow (1) with nonnegative holomorphic bisectional curvature 
and positive Ricci curvature is necessarily a translating Kähler-Ricci soliton. Furthermore, if $X$ is simply connected, then $g$ is a gradient soliton.

The proof of Proposition 3.1 follows from our Li-Yau-Hamilton estimate $[1,3]$ (also called differential Harnack estimate) for the Kähler-Ricci flow and the strong maximum principle argument.

In complex dimension one there is only one (up to scaling) translating KRS of positive curvature, called cigar soliton, found by Hamilton [10]. The metric is defined on the complex plane $\mathbf{C}$ and can be written explicitly, at $t=0$, as

$$
d s^{2}=\frac{|d z|^{2}}{1+|z|^{2}}
$$

The cigar soliton has maximal curvature at the origin and is asymptotic to a flat cylinder at the infinity. Later in [2], the author found for each $n \geq 2$ a translating KRS on $\mathbf{C}^{n}$, invariant under the unitary group and of positive sectional curvature. Furthermore, this rotationally symmetric soliton has the following geometric properties: curvature at geodesic distance $s$ from the origin decays like $1 / s$; while volume of geodesic ball of radius $s$ centered at the origin grows like $s^{n}$. In general, the special nature of translating KRSs also allow us to draw some very nice conclusions about both its complex analytic and geometric properties:

Proposition 3.2 (Cao-Hamilton [4]) Let $\left(X^{n}, g\right)$ be a translating gradient $K R S$ with positive Ricci curvature such that the scalar curvature $R$ assumes its maximum 1 in space-time. Let $f$ be a potential function of $g$. Then $f$ is a strictly convex exhaution function on $X$. In particular, $X$ is a Stein manifold diffeomorphic to $R^{2 n}$.

A fact important in the proof of Proposition 3.2 is that the potential function $f$ satisfies the equation

$$
R+|D f|^{2}=1 .
$$

A straightforward modification of a result of Hamilton (Theorem 20.2 in [13]) yields

Proposition 3.3 Let $\left(X^{n}, g\right), n \geq 2$, be a translating KRS with bounded and nonnegative bisectional curvature such that the scalar curvature $R$ assumes its maximum in space-time. For any (small) constant $\epsilon>0$, if there exists a positive constant $C_{\epsilon}$ such that the $(1+\epsilon)$-asymptotic scalar curvature ratio

$$
A_{1+\epsilon}=\lim \sup _{s \rightarrow \infty} R(x) s^{1+\epsilon}(x)<C_{\epsilon},
$$


then $g$ must be flat. In particular, if $g$ is non-flat, then $A_{1+\epsilon}=\infty$ for any $\epsilon>0$.

Note in particular the asymptotic curvature ratio $A \equiv A_{2}$ must be infinite for any translating KRS of nonnegative bisectional curvature.

\section{The proof of main results.}

Proof of Theorem 1.1: Let $\left(X^{2}, g\right)$ be a Type II singularity model as in the statement of Theorem 1.1. For simplicity we assume $X^{2}$ is simply connected, otherwise we can replace it by its universal cover. First we claim the Ricci curvature of $g$ must be strictly positive at all points and all time. If the Ricci curvature is not strictly positive at some point $x_{0}$ and some time $t_{0}$, then by the splitting theorem $2.1,\left(X^{2}, g\right)$ splits as a quotient of a product of the flat complex plane $\mathbf{C}$ with a complete Riemann surface $\Sigma$ of positive Gaussian curvature. Note $g$ restricted to $\Sigma$ remains a Type II singularity model satisfying the local injectivity radius estimate. But the only Type II singularity model on a Riemann surface with positive Gaussian curvature is the cigar soliton, which does not satisfy the local injectivity radius estimate. A contradiction. Therefore the Ricci curvature of $g$ is strictly positive everywhere.

Now, it follows from Proposition 3.1 that the Type II limit solution $\left(X^{2}, g\right)$ is a translating gradient Kähler-Ricci soliton with bounded nonnegative holomorphic bisectional curvature and positive Ricci curvature. Then by Proposition 3.3, we know that asymptotic scalar curvature ratio $A=A_{2}<\infty$. Therefore, we can apply Lemma 22.2 of Hamilton [13] to find a sequence of points $x_{j} \in X$ going to infinity at time $t=0$, a sequence of radii $r_{j}>0$, and a sequence of positive numbers $\delta_{j} \rightarrow 0$ such that

(a) $R(x, 0) \leq\left(1+\delta_{j}\right) R\left(x_{j}, 0\right)$ for all $x$ in the ball $B_{r_{j}}\left(x_{j}, 0\right)$ of radius $r_{j}$ around $x_{j}$ at time $t=0$;

(b) $r_{j}^{2} R\left(x_{j}, 0\right) \rightarrow \infty$;

(c) if $s_{j}$ is the distance of $x_{j}$ from some origin $O$ at time $t=0$, then $\lambda_{j}=s_{j} / r_{j} \rightarrow \infty$

(d) the balls $B_{r_{j}}\left(x_{j}, 0\right)$ are disjoint.

We can then blow down $\left(X^{2}, g\right)$ as in [13] by taking a sequence of dilations of $\left(X^{2}, g\right)$ around the sequence of points $x_{j}$ which we take as our new origins $O_{j}$, and we shrink down instead of expanding to make the scalar curvature 
$R\left(x_{j}, 0\right)$ dilate to equal 1 at $\left(O_{j}, 0\right)$. The balls $B_{r_{j}}\left(x_{j}, 0\right)$ are then dilated to the balls centered at the origin $O_{j}$ of radii $\hat{r}_{j}=r_{j}^{2} R\left(x_{j}, 0\right) \rightarrow \infty$ by property (b). Property (a) gives good bounds on the curvature in these balls at time $t=0$, while the same bounds for $t \leq 0$ follows from the fact that the scalar curvature $R(x, t)$ of $g$ is pointwise increasing in time, a consequence of our Li-Yau type estimate for $R(x, t)$ (see $[1,3]$ ). The local injectivity radius estimate (2) now becomes

$$
\operatorname{inj}_{B_{r_{j}}}\left(O_{j}, g\right) \geq \frac{\beta}{\sqrt{R\left(O_{j}, 0\right)}}
$$

since it is invariant under dilation.

Now we have everything we need to take a limit of the dilations of $\left(X^{2}, g\right)$ around the points $\left(x_{j}, 0\right)$, dilating time like distance squared and keeping $t=0$ as $t=0$ in the new limit, which is denoted by $\left(\widehat{X}^{2}, \widehat{g}\right)$, where $\widehat{g}$ is a complete solution to the Kähler-Ricci flow (1) on the limiting noncompact complex surface $\widehat{X}^{2}$ with the limiting origin point $\widehat{O}$ and an ancient time interval $-\infty<t \leq \Omega$, for some $\Omega>0$ by Shi's short time existence result [17], and with bounded and nonnegative holomorphic bisectional curvature such that the scalar curvature $\widehat{R}(x, t) \leq 1$ everywhere for $t \leq 0$ and $\widehat{R}(\widehat{O}, 0)=1$ at the origin at time $t=0$.

The next step is to produce a Ricci-flat direction at $\widehat{O}$ in the blow-down limit $\left(\widehat{X}^{2}, \widehat{g}\right)$. First we prove a proposition, which is of independent interest and will be important in showing the Ricci tensor $\widehat{R}_{i \bar{j}}$ of $\widehat{g}$ must have a null vector at $\widehat{O} \in \widehat{X}^{2}$.

Proposition 4.1 Let $\Theta$ denote the second Chern form of $\left(X^{2}, g\right)$ at $t=0$. Then, the second Chern number integral

$$
c_{2}(X)=\int_{X} \Theta
$$

of $\left(X^{2}, g\right)$ is well defined. In fact, $c_{2}(X) \leq 1$.

Proof: It is well-known that for any Kähler surface of nonnegative holomorphic bisectional curvature, the second Chern form, or equivalently the Gauss-Bonnet-Chern integrand, $\Theta$ of $\left(X^{2}, g\right)$ is pointwise nonnegative. In fact, $\Theta$ can be expressed explicitly as

$$
\begin{aligned}
\Theta & =\frac{1}{8 \pi^{2}}\left(R^{2}+|R m|^{2}-2|R c|^{2}\right) d \mu \\
& =\frac{1}{4 \pi^{2}}\left(R_{1 \overline{1} 1 \overline{1}} R_{2 \overline{2} 2 \overline{2}}+2 R_{1 \overline{1} 2 \overline{2}}^{2}+\left|R_{1 \overline{2} 1 \overline{2}}\right|^{2}+2\left|R_{1 \overline{1} 1 \overline{2}}\right|^{2}+2\left|R_{2 \overline{2} 1 \overline{2}}\right|^{2}\right) d \mu .
\end{aligned}
$$


Here $d \mu$ is the volume form of $g$ at $t=0$. Thus, in order to prove $c_{2}(X)$ is well defined, it suffices to prove the integrals $c_{2}\left(U_{i}\right)=\int_{U_{i}} \Theta$ is uniformly bounded for some exhaution sequence of subsets $U_{i}$ of $X^{2}$.

Since $\left(X^{2}, g\right)$ is a gradient KRS, by Proposition 3.2, there exists a strictly convex exhaustion potential function $f$ on $X$. Taking $U_{i}=\{f \leq i\}$, then as is well-known, we have

$$
c_{2}\left(U_{i}\right)=\int_{U_{i}} \Theta=\chi\left(U_{i}\right)+\{\text { boundary contribution }\}
$$

where $\chi\left(U_{i}\right)$ denotes the Euler characteristic of the set $U_{i}$. Now $\partial U_{i}$ has positive definite second fundamental form and it can be shown (see e.g. Section 4 in [8]) that the boundary term above is nonpositive. Therefore

$$
c_{2}\left(U_{i}\right) \leq \chi\left(U_{i}\right)
$$

But by Proposition 3.2, $X$ is diffeomorphic to $\mathbf{R}^{4}$ and each $U_{i}$ is diffeomorphic to the unit ball. Thus $\chi\left(U_{i}\right)=1$ for all $i$ which implies $c_{2}(X) \leq 1$.

Now we can prove

Lemma 4.2 The Ricci tensor of $\widehat{g}$ must have a zero eigenvalue at the origin $\widehat{O} \in \widehat{X}^{2}$ at time $t=0$.

Proof of Lemma 4.2: We prove by contradiction. Suppose Lemma 4.2 is not true. Then before taking the blow-down limit, there must exist a positive number $\varepsilon>0$ and a subsequence, again indexed by $j$, of $\left\{x_{j}\right\}$ in $X$ such that

$$
\varepsilon_{j}=\frac{\sigma_{j}}{R\left(x_{j}, 0\right)} \geq \varepsilon \quad \text { for all } \quad j=1,2, \cdots,
$$

where we denote by $\sigma_{j}$ the minimum of the Ricci curvature of $g$ at $x_{j}$ at $t=0$.

We are going to show this leads to a contradiction by closely examing the relation between Chern numbers $c_{2}$ and $c_{1}^{2}$.

On one hand, by the local derivative estimate of Shi [17] (or Theorem 13.1 in [13]) and properties (a) and (b), we have at $t=0$ the estimate

$$
\begin{aligned}
\sup _{x \in B_{r_{j}}\left(x_{j}, 0\right)}|\nabla R m(x, 0)|^{2} & \leq C R^{2}\left(x_{j}, 0\right)\left(\frac{1}{r_{j}^{2}}+R\left(x_{j}, 0\right)\right) \\
& \leq 2 C R^{3}\left(x_{j}, 0\right)
\end{aligned}
$$

where $R m$ is the curvature tensor of $g$ and $C>0$ is a universal constant. 
For any $x \in B_{r_{j}}\left(x_{j}, 0\right) \subset X$, we obtain from (5) and (6) that the minimum of the Ricci curvature $\sigma_{\min }(x)$ at $x$, satisfies

$$
\begin{aligned}
\sigma_{\min }(x) & \geq \sigma_{j}-\sqrt{2 C} R^{3 / 2}\left(x_{j}, 0\right) d_{0}\left(x, x_{j}\right) \\
& \geq R\left(x_{j}, 0\right)\left(\varepsilon-\sqrt{2 C} \cdot \sqrt{R\left(x_{j}, 0\right)} \cdot d_{0}\left(x, x_{j}\right)\right) \\
& \geq \frac{\varepsilon}{2} R\left(x_{j}, 0\right)
\end{aligned}
$$

whenever

$$
d_{0}\left(x, x_{j}\right) \leq l_{j}=: \frac{\varepsilon}{2 \sqrt{2 C} \cdot \sqrt{R\left(x_{j}, 0\right)}} .
$$

Thus, from property (a) and (7), there exists $j_{0}>0$ such that for any $j \geq j_{0}$ and $x \in B_{l_{j}}\left(x_{j}, 0\right)$, we have the estimate

$$
\frac{\varepsilon}{2} R\left(x_{j}, 0\right) \leq \sigma_{\min }(x) \leq 2 R\left(x_{j}, 0\right)
$$

in the geodesic ball $B_{l_{j}}\left(x_{j_{k}}, 0\right)$.

Hence

$$
R c_{g}{ }^{2}(x) \geq\left(\frac{\varepsilon}{2}\right)^{2} R^{2}\left(x_{j}, 0\right) d \mu
$$

for all $x \in B_{l_{j}}\left(x_{j}, 0\right)$.

Therefore,

$$
\begin{aligned}
\sum_{j=j_{0}}^{\infty} \int_{B_{l_{j}}\left(x_{j}, 0\right)} R c_{g}^{2} & \geq C(\varepsilon) \sum_{j=j_{0}}^{\infty} R^{2}\left(x_{j}, 0\right) \cdot C_{1}\left(\frac{\varepsilon}{2 \sqrt{2 C} \cdot \sqrt{R\left(x_{j}, 0\right)}}\right)^{4} \\
& =C(\varepsilon) \sum_{j=j_{0}}^{\infty} \frac{C_{1} \varepsilon^{4}}{C^{2}} \\
& =+\infty
\end{aligned}
$$

On the other hand, if we diagonalize the Ricci tensor at each point $x$, we have

$$
\begin{aligned}
R c_{g}{ }^{2}(x) & =\frac{1}{\pi^{2}} R_{1 \overline{1}}(x) R_{2 \overline{2}}(x) d \mu \\
& =\frac{1}{\pi^{2}}\left(R_{1 \overline{1} 1 \overline{1}} R_{2 \overline{2} 2 \overline{2}}+R_{1 \overline{1} 1 \overline{1}} R_{1 \overline{1} 2 \overline{2}}+R_{1 \overline{1} 2 \overline{2}}^{2}+R_{1 \overline{1} 2 \overline{2}} R_{2 \overline{2} 2 \overline{2}}+R_{2 \overline{2} 2 \overline{2}}^{2}\right) d \mu .
\end{aligned}
$$

From (4), (5), and (10), it is easy to check that there exists a constant $C^{\prime}(\epsilon)$ such that

$$
R c_{g}^{2}\left(x_{j}\right) \leq C^{\prime}(\epsilon) \Theta\left(x_{j}\right)
$$


for all $j$. It then follows that

$$
\sum_{j=j_{0}}^{\infty} \int_{B_{l_{j}}\left(x_{j}, 0\right)} R c_{g}^{2} \leq C^{\prime}(\epsilon) \sum_{j=j_{0}}^{\infty} \int_{B_{l_{j}}\left(x_{j}, 0\right)} \Theta<C^{\prime}(\epsilon) c_{2}(X)<C^{\prime}(\epsilon),
$$

which contradicts (9). Thus the proof of Lemma 4.2 is completed.

Now, it follows from Lemma 4.2 and Theorem 2.1 that, since $\widehat{g}$ is nonflat, the universal cover of $\widehat{X}^{2}$ splits holomorphically isometrically as a product of the flat complex plane $\mathbf{C}$ and a Riemann surface $\Sigma$ of positive Gaussian curvature. The factor $\Sigma$ may not be yet of Type I or Type II, but we can take a further limit of dilation, as in [13], also by shrinking, to get yet another limit $N$ which will be either of Type I or Type II. However, $N$ cannot be Type II, because otherwise $N$ has to be the cigar soliton, but cigar soliton does not satisfy the local injectivity radius estimate. So $N$ must be of Type I. Then by a result of Hamilton [13] (Theorem 26.1) the only Type I ancient solutions to the Ricci flow on a Riemann surface which are complete with bounded curvature are the (round) Riemann sphere, or the flat complex plane $\mathbf{C}$ and its quotients. Since $N$ has positive curvature, it must be the Riemann sphere $\mathbf{C P}^{1}$.

Now a sequence of dilations of $\left(X^{2}, g\right)$ converges to a quotient of $\left(\widehat{X}^{2}, \widehat{g}\right)$, which splits into $\mathbf{C} \times \Sigma$, and a sequence of dilations of $C \times \Sigma$ converges to $\mathbf{C} \times C P^{1}$. Again, since a dilation of a dilation is a dilation, and a limit of limits is a limit by picking an appropriate subsequence. Thus a limit of dilations of $\left(X^{2}, g\right)$ converges to $\mathbf{C} \times C P^{1}$.

Proof of Corollary 1.1: Suppose we have a 2-dimensional Type II singularity model $\left(X^{2}, g\right)$ with $\nu_{X}>0$. Using the convex exhaustion function $f$ in Proposition 3.2 and modifying an argument of Cheeger-Gromov-Taylor [5], it follows that the local injectivity radius estimate (ii) holds for $\left(X^{2}, g\right)$. Hence, according to Theorem 1.2, a sequence of dilations of $g$ converges to a limit $\widehat{g}$ on some noncompact complex manifold $\widehat{X}$. Furthermore $\left(\widehat{X}^{2}, \widehat{g}\right)$ is a quotient of the product $\mathbf{C} \times \mathbf{C P}^{1}$ of flat complex plane $\mathbf{C}$ and the complex projective plane $\mathbf{C P}^{1}$ so that $\nu_{\widehat{X}}=0$. On the other hand, we must have $\nu_{\widehat{X}}>0$ since the condition of having Euclidean volume growth is clearly preserved under dilations and taking limits. A contradiction.

Proof of Theorem 1.2: We can proceed similarly as in the proof of Theorem 1.1. Let $g$ be the ancient solution to the Kähler-Ricci flow (1) satisfying all the assumptions in Theorem 1.2. Since the asymptotic scalar curvature ratio $A=\infty$, we can apply Lemma 22.2 of Hamilton [13] and get blow-down limit $\left(\widehat{X}^{n}, \widehat{g}\right)$ as in the proof of Theorem 1.1. 
Lemma 4.3 The Ricci tensor of $\widehat{g}$ must have a zero eigenvalue at the origin $\widehat{O} \in \widehat{X}^{n}$ at time $t=0$.

Proof of Lemma 4.3: We again prove by contradiction. Suppose Lemma 4.3 is not true so that condition (5) in the proof of Lemma 4.2 holds. We are going to show this leads to a contradiction to assumption (iii).

On one hand, as in the proof of Lemma 4.2, we have the estimate

$$
\frac{\varepsilon}{2} R\left(x_{j}, 0\right) \leq \sigma_{\min }(x) \leq 2 R\left(x_{j}, 0\right)
$$

in the geodesic ball $B_{l_{j}}\left(x_{j_{k}}, 0\right)$ and hence

$$
R c_{g}{ }^{n}(x) \geq\left(\frac{\varepsilon}{2}\right)^{n} R^{n}\left(x_{j}, 0\right) d \mu
$$

for all $x \in B_{l_{j}}\left(x_{j}, 0\right)$. Here $d \mu$ is the volume form of $g$ at $t=0$. It then follows that

$$
\begin{aligned}
c_{1}^{n}(X) & \geq \sum_{j=j_{0}}^{\infty} \int_{B_{l_{j}}\left(x_{j}, 0\right)} R c_{g}{ }^{n} \\
& \geq C(\varepsilon) \sum_{j=j_{0}}^{\infty} R^{n}\left(x_{j}, 0\right) \cdot C_{1}\left(\frac{\varepsilon}{2 \sqrt{2 C} \cdot \sqrt{R\left(x_{j}, 0\right)}}\right)^{2 n} \\
& =C^{\prime}(\varepsilon) \sum_{j=j_{0}}^{\infty} \frac{C_{1} \varepsilon^{2 n}}{C^{n}} \\
& =+\infty
\end{aligned}
$$

which is a contradiction to assumption (iii), and the proof of Lemma 4.3 is completed.

Now the blow-down limit $\left(\widehat{X}^{n}, \widehat{g}\right)$ has a Ricci-flat direction, hence splitting theorem 2.1 applies and the universal cover of $\widehat{X}$ splits holomorphically isometrically as a product of flat $\mathbf{C}^{l}$ and a factor $N^{n-l}$ of nonnegative bisectional curvature and positive Ricci curvature. The factor $N$ may not be yet of Type I or Type II, but we can take a further limit of dilation, as in [11], also by shrinking, to get yet another limit $\tilde{N}$ which will be either of Type I or Type II. If $\tilde{N}$ is of Type I, or Type II with $c_{1}^{n-l}(\tilde{N})=\infty$, then we are done. If $\tilde{N}$ is of Type II but with $c_{1}^{n-l}(\tilde{N})<\infty$, then we can repeat the dimension reduction process above to split out more flat factors until we arrive at some factor $\widehat{N}^{n-k}(k \geq l)$ which is either of Type I, or of Type II with $c_{1}^{n-k}(\widehat{N})=\infty$. 
Now a sequence of dilations of $\left(X^{n}, g\right)$ converges to a quotient of $\left(\widehat{X}^{n}, \widehat{g}\right)$, which splits into $\mathbf{C}^{l} \times N^{n-l}$, and a sequence of dilations of $\mathbf{C}^{l} \times N^{n-l}$ converges to $\mathbf{C}^{l} \times \tilde{N}^{n-l}$, and a sequence of dilations of $\mathbf{C}^{l} \times \tilde{N}^{n-l}$ converges to $\mathbf{C}^{k} \times$ $\widehat{N}^{n-k}$. Since a dilation of a dilation is a dilation, and a limit of limits is a limit by picking an appropriate subsequence. Thus a limit of dilations of $\left(X^{n}, g\right)$ converges to $\mathbf{C}^{k} \times \widehat{N}^{n-k}$, where $\widehat{N}^{n-k}$ is either of Type I, or of Type II with $c_{1}^{n-k}(\widehat{N})=\infty$.

\section{References.}

[1] H.-D. Cao, On Hanarck's inequalities for the Kähler-Ricci flow, Invent. Math. 109 (1992), 247-263.

[2] H.-D. Cao, Existence of gradient Kähler-Ricci solitons, Elliptic and Parabolic Methods in Geometry, (Minneapolis, MN, 1994) B. Chow, R. Gulliver, S. Levy, J. Sullivan ed., AK Peters (1996) 1-16.

[3] H.-D. Cao, Limits of solutions to the Kähler-Ricci flow, J. Differ. Geom., 45 (1997), 257-272.

[4] H.-D. Cao and R. S. Hamilton, Gradient Kähler-Ricci solitons and periodic orbits, Comm. Anal. Geom., 8 (2000), 517-529.

[5] J. Cheeger, M. Gromov, and M. Taylor, Finite propagation speed, kernal estimates for functions of the Laplace operator, and the geometry of complete Riemannian manifold, J. Differ. Geom., 17 (1982), 15-53.

[6] B.-L. Chen, S.-H. Tang \& X.-P. Zhu, Uniformization of complete noncompact surfaces with positive bisectional curvature, preprint.

[7] B.-L. Chen \& X.-P. Zhu, A property of Kähler-Ricci solitons on complete complex surfaces, Geometry and Nonlinear Partial Differential Equations, AMS/IP Studies in Advanced Mathematics, 29 (2002), 5-12.

[8] R. E. Greene and $\mathrm{H}$. Wu, $C^{\infty}$ convex functions and manifolds of positive curvature, Acta. Math. 137 (1976), 209-245.

[9] R.S. Hamilton, Four-manifolds with positive curvature operator, J. Differ. Geom. 24 (1986), 153-179.

[10] R. S. Hamilton, The Ricci flow on surfaces, Mathematics and General Relativity, Contemporary Mathematics, 71 (1988), 237-261. 
[11] Hamilton, R.S., The Harnack estimate for the Ricci flow, J. Differ. Geom. 37 (1993), 225-243.

[12] R. S. Hamilton, Eternal solutions to the Ricci flow, J. Differ. Geom. 38 (1993), 1-11.

[13] R. S. Hamilton, Formation of singularities in the Ricci flow, Surveys in Diff. Geom. 2 (1995) 7-136, International Press, Boston.

[14] S. Kobayashi and K. Nomizu, Foundations of Differential Geometry, Wiley, New York, Vol.II, 1969.

[15] N. Mok, The uniformization theorem for compact Kähler manifolds of nonnegative holomorphic bisectional curvature, J. Differ. Geom. 27 (1988), 179-214.

[16] L. Ni and L.-F. Tam, Liouville properties of plurisubharmonic functions, preprint.

[17] W.-X. Shi, Ricci flow and the uniformization on complete noncompact Kähler manifolds, J. Differ. Geom. 45 (1997), 94-220.

Department of Mathematics

LEHIGH UNIVERSITY

BeTHLEHEM, PA 18015

huc2@lehigh.edu

$\&$

Department of Mathematics

TEXAS A\&M UNIVERSITY

College Station, TX 77843

cao@math.tamu.edu 